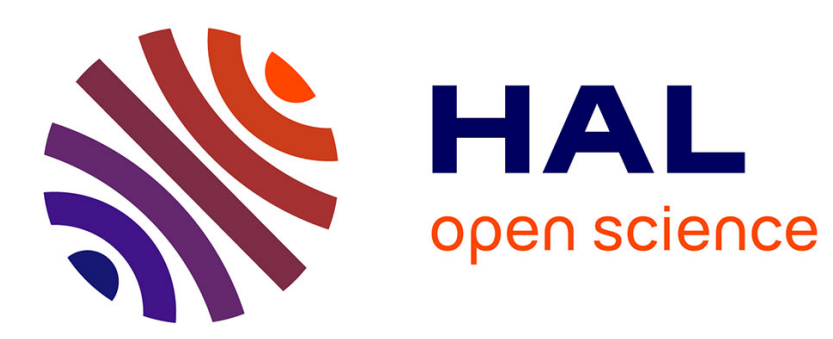

\title{
Modélisation de l'impédance acoustique normale des structures à panneaux perforés
}

\author{
J. Ducourneau, P. Ozouf
}

\section{To cite this version:}

J. Ducourneau, P. Ozouf. Modélisation de l'impédance acoustique normale des structures à panneaux perforés. Journal de Physique IV Proceedings, 1994, 04 (C5), pp.C5-113-C5-116. 10.1051/jp4:1994518 . jpa-00252861

\section{HAL Id: jpa-00252861 https://hal.science/jpa-00252861}

Submitted on 1 Jan 1994

HAL is a multi-disciplinary open access archive for the deposit and dissemination of scientific research documents, whether they are published or not. The documents may come from teaching and research institutions in France or abroad, or from public or private research centers.
L'archive ouverte pluridisciplinaire HAL, est destinée au dépôt et à la diffusion de documents scientifiques de niveau recherche, publiés ou non, émanant des établissements d'enseignement et de recherche français ou étrangers, des laboratoires publics ou privés. 


\section{Modélisation de l'impédance acoustique normale des structures à panneaux perforés}

\section{J. DUCOURNEAU et P. OZOUF}

Laboratoire d'Acoustique de Métrologie d'Instrumentation, 38 rue des 36 Ponts, 31400 Toulouse cedex, France

Abstract: Perforated facings are currently used in sound absorption applications.

Different kinds of these facings are used in acoustical treatment of halls, in silencers and in aerial navigation. Our purpose is to study the acoustic impedance of these facings. One of the particular types of absorbing materials that will be considered in this paper consists of a porous homogenous blanket or an air layer placed in front of a hard wall and covered with a perforated facing. In another way, the air layer between the perforated facing and the wall may be divided into cells by means of partitions.

Our study is based on Brillouin's theory relating to perforated facings and on works carried out by Morse and Ingard concerning porous material.The choosen model for simulating these different profiles has been verified practicaly using a Kundt tube.

\section{Introduction}

Les principales configurations existant dans le milieux industriels sont de deux types : structure continue - structure cloisonnées, elles comportent toutes les deux une parois perforée d'épaisseur $h$, elles sont placées à une distance $e$ du mur et elles diffèrent par la nature de cet espace laminaire qui sépare cette cloison du mur.

Dans les deux structures, l'espace laminaire se comporte comme une cavité de résonateur de Helmholtz, et chaque trou de la parois perforée s'identifie à un col. Nous avons, dans un premier temps, développé une étude sur le résonateur de Helmholtz puis nous avons étendu celle-ci aux différentes structures. Le matériau poreux susceptible de remplir cet espace laminaire sera caractérisé par des paramètres précis avec lesquels nous pourrons déterminer la constante de propagation et son impédance caractéristique.

\section{Résonateur de Helmholzt}

L'étude expérimentale du résonateur de Helmholtz se fera essentiellement au tube de Kundt. La géométrie de notre plan d'étude sera la suivante : un tuyau de section $S$ dans lequel vont se propager des ondes planes, un col de résonateur que l'on assimilera à un tuyau étroit de section $s$, puis une cavité de section $S$. Nous allons supposer l'existence d'une onde longitudinale se propageant parallèlement à l'axe Ox, et mettant en évidence les mouvements d'air longitudinaux dans la cavité.

$$
\Theta=e^{j \omega t}\left(I . e^{\frac{j \omega x}{c}}+R \cdot e^{\frac{-j \omega x}{c}}\right) I \text { et } R \text { amplitude de l'onde incidente et réfléchie }
$$

en $\mathrm{x}=+e$ l'impédance réduite par rapport à l'impédance de l'air : $z_{1}=-j \cot (k e)$ 
avec le changement de section : $z_{2}=-j \frac{s}{S} \cot (k e)$

Si nous ramenons $z_{2}$ en $z_{3}$, il faut faire apparaître un terme résistif $\mathrm{R}$ dû à des forces de frottements $\mathrm{du}$ volume d'air qui vibre dans le col du résonateur.

$z_{3}=R+j\left(\frac{\tan \left(k l^{\prime}\right)-\frac{s}{S} \cot (k e)}{1+\frac{s}{S} \tan \left(k l^{\prime}\right) \cot (k e)}\right)$

avec l'=longueur corrigée du col dans lequel la masse d'air vibre.

Une étude plus approfondie sur la valeur de cette longueur corrigée a été développée plus loin.

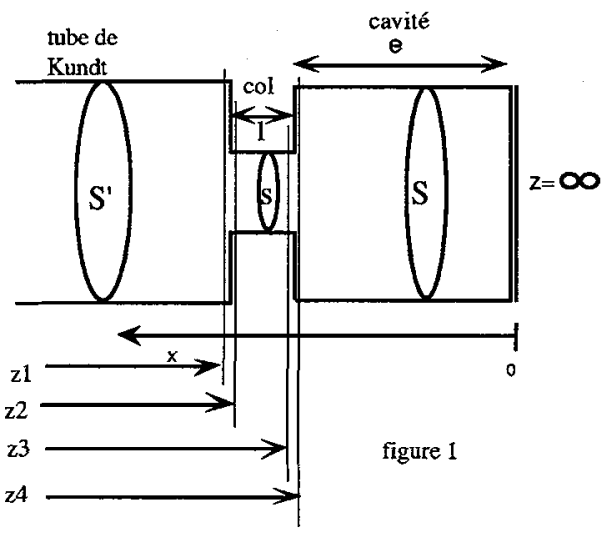

Enfin le nouveau changement de section intervient de $z_{3}$ à $z_{4}$ :

Simplification : $\mathrm{Si}$ on considère que la cavité est petite devant la longueur d'onde (Ceci sera d'autant plus vérifié si

$$
z_{4}=\frac{S^{\prime}}{S} R+j \frac{S^{\prime}}{s}\left(\frac{\tan \left(k l^{\prime}\right)-\frac{s}{S} \cot (k e)}{1+\frac{S}{S} \tan \left(k l^{\prime}\right) \cot (k e)}\right)
$$

les fréquences sont basses) : $e<\frac{\lambda}{4}$, en négligeant le volume du col devant celui de la cavité et en multipliant par $\rho c$, on voit apparaître un terme de masse dû au déplacement d'air dans le col, puis un terme d'élasticité.

A la résonance de la cavité : $\omega_{0}=c \sqrt{\frac{s}{v l}}$

\subsection{La longueur corrigée}

$$
z_{4}=R_{e}+j \frac{S^{\prime}}{s}\left[\rho \omega{ }^{2}-\frac{\rho s c^{2}}{\omega V}\right]
$$

Si l'orifice du col du résonateur débouche de part et d'autre à l'air libre, le piston-plan d'air contenu dans ce col rayonne. La masse d'air entraînée n'est pas $m$ mais $m^{\prime}$ : cette différence se traduit par une longueur corrigée du col $l=l+\delta$. L'impédance de rayonnement d'un piston-plan est définie par :

$Z_{r a}=\frac{\bar{p}}{\int \vec{u} \vec{n} \cdot d s}$ On montre que $Z_{r a}=\rho c\left[1-\frac{J_{1}(2 k r)}{k r}\right]+j\left[\frac{H_{1}(2 k r)}{k r}\right] H_{1}$ : fonction de Struve du 1 ordre.

où le terme imaginaire est un terme de masse d'air entraînée à proximité du col.

en approximant $j \frac{H_{1}(2 k r)}{k r} \approx j \frac{8}{3 \pi} k r \rho c \approx j \rho c \frac{\omega}{c} \frac{8 r}{3 \pi}=j \rho \omega l^{\prime}$ d'où $l^{\prime}=\frac{8 r}{3 \pi}$

Ingard [2] distingue une longueur corrigée intérieure plus précise, calculée à partir d'une ouverture circulaire rayonnant sur un cylindre infinie. Si le taux de perforation $\sigma<0.4$ alors $l^{\prime}=0.48 \sqrt{S}(1-1.3 \sqrt{\sigma})$

\subsection{Dissipation d'énergie dans un résonateur.}

La dissipation d'énergie, causée par les frottements d'un piston d'air dans un col, est donnée par : $W=\frac{1}{2} \int_{S} R_{S} U_{S}^{2} d S$ où $U_{S}$ est l'amplitude de la vitesse vibratoire tangentielle du piston dans le col, $S$ la surface et $R_{\mathcal{S}}$ "la résistance visqueuse de surface ".

Ingard [2] souligne l'existence d'une couche limite visqueuse à l'intérieur du col dans laquelle l'air vibre librement. Au dessus de celle-ci se trouve un anneau d'air figé d'une certaine épaisseur qui ne participe pas à la vibration "effet de peau". En calculant la dissipation d'énergie à la périphérie du conduit étroit et sur les 
deux ouvertures on peut déterminer la résistance acoustique spécifique traduisant cette dissipation en fonction de la fréquence $f$ $R=1.710^{-3}\left(\frac{h}{r}+2\right) \sqrt{f} \frac{1}{\rho c}$

\section{Structure cloisonnées}

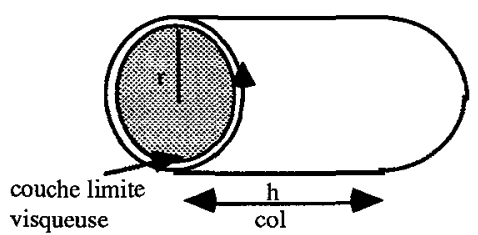

L'arrivée d'ondes planes d'incidence $\theta$ sur la paroi perforée donne naissance à un mouvement d'air parallèle à l'axe Ox. L'excitation extérieure donne une vibration déphasée d'une alvéole à l'autre. On a donc dans une alvéole :

$$
\Theta=e^{j \omega \frac{y \sin \theta}{c}}\left(I e^{\frac{j \omega x}{c}}+\operatorname{Re} \frac{-j \omega x}{c}\right) e^{j \omega t} . \text { Le terme } e^{j \omega \frac{y \sin \Theta}{c}} \text { va }
$$

disparaître dans le rapport $\frac{p}{u}$ par conséquent l'impédance acoustique normale d'une structure nid d'abeille ne dépendra pas de l'angle d'incidence de l'onde plane extérieure.

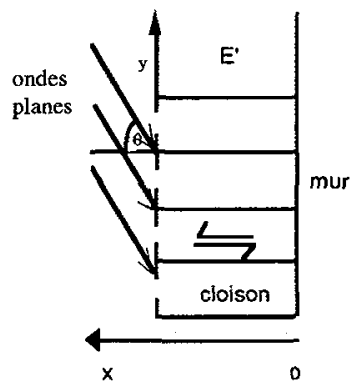

On pourra écrire comme pour le résonateur :

$z=\frac{R}{\sigma}+j\left[\frac{k l^{\prime}-\frac{\sigma}{\mu k e}}{1+\frac{\sigma l^{\prime}}{\mu e}}\right]$ avec $\mu=\frac{n S}{S^{\prime}}:$ rapport de l'aire des alvéoles sur l'aire totale $; \sigma=\frac{n s}{S^{\prime}}:$ taux de perforation

\section{Structure continue}

\subsection{L'espace laminaire contient de l'air}

Dans l'espace $E^{\prime}$ compris entre le mur et la paroi perforée le potentiel des vitesses a la même forme que dans l'espace $E$ à l'avant de la tôle perforée :

$$
\Theta=e^{j \omega \frac{y \sin (\theta)}{c}}\left(I e^{j \omega \frac{x \cos (\theta)}{c}}+\mathrm{Re}^{j \omega \frac{-x \cos (\theta)}{c}}\right) e^{j \omega t}
$$

Par unité de surface nous obtenons :

$$
z=\frac{R}{\sigma}+j \frac{1}{\sigma}\left[k(h+\delta)-\frac{\cot (k e \cos (\theta))}{\cos (\theta)}\right]
$$

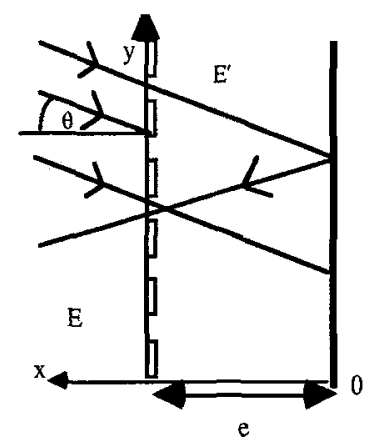

\subsection{L'espace laminaire contient un matériau poreux}

Afin d'étudier l'impédance acoustique d'un matériau poreux, on va déterminer son impédance caractéristique $z_{1}$, puis sa constante de propagation $k_{1}$. Pour cela nous allons réécrire les équations de propagation dans le matériau poreux en nous appuyant sur la théorie d'Ingard [1].

Celle-ci nécessite la connaissance de paramètres qui caractérisent ce matériau : la porosité $P$, la résistance au passage de l'air $r$, le facteur de structure $m$. Les équations de continuité et de la dynamique deviennent

$$
\operatorname{div}(\vec{u})=-\frac{P q}{\rho c^{2}} \frac{\partial p}{\partial t} ; \rho m \frac{\partial \vec{u}}{\partial t}+r \vec{u}=-\operatorname{grad}(p) \Rightarrow k_{1}=\sqrt{P q} \frac{\omega}{c} \sqrt{\left[m-j \frac{r}{\rho \omega}\right]} \quad \text { et } \quad \mathrm{Z}_{1}=\rho c \sqrt{\left(\mathrm{m}-\mathrm{j} \frac{\mathrm{r}}{\rho \omega}\right) / \sqrt{\mathrm{Pq}}}
$$

L'impédance acoustique normale ramenée à l'avant du matériau poreux est égale à : $Z=-j Z_{1} \cot \left(k_{1} e\right)$.

Si on rajoute une tôle perforée à l'avant du matériau, il faut ajouter un terme de masse causé par la face intérieure de la tôle perforée sur le matériau poreux puis un terme de masse due à la face extérieure de la tôle perforée : Par unité de surface : $z=\delta \frac{\mathrm{r}}{\rho \mathrm{c}} \frac{1}{\sigma}+\mathrm{j} \frac{1}{\sigma}\left[\mathrm{k}(\mathrm{h}+(1+\mathrm{m}) \delta)-\frac{\mathrm{Z}_{1}}{\rho c} \cot \left(\mathrm{k}_{1} \mathrm{e}\right) . \sigma\right]$ 


\section{Résultats}

Nous comparons içi les résultats obtenus avec l'expérience réalisée au tybe de Kundt pour une structure cloisonnées, une mousse poreuse et cette même mousse avec une tôle perforées placée à l'avant.

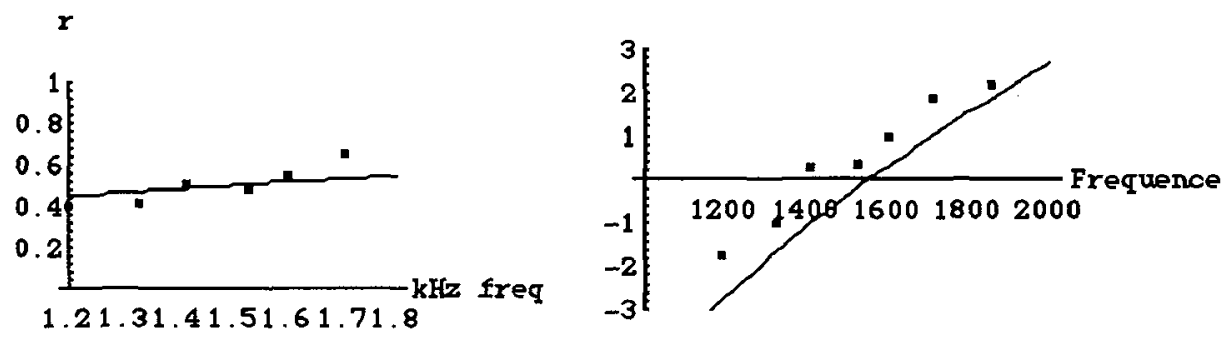

Structure cloisonnée $\sigma=4.43 \% \quad h=1 \mathrm{~mm} \quad \mu=0.34$

Partie reelle reduite

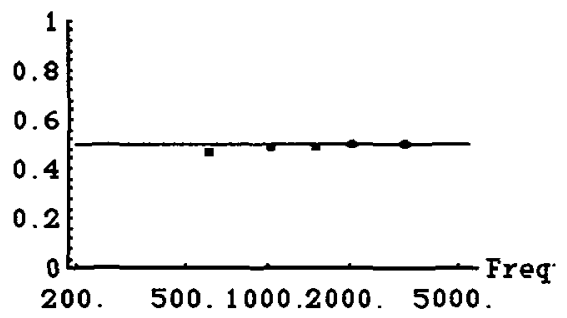

mousse poreuse $e=25 \mathrm{~mm}$
Partie imaginaire reduite

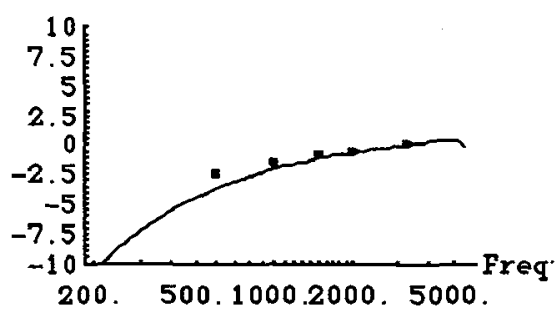

$r=12550 \mathrm{~kg} / \mathrm{sm}^{3} \quad m=1.37 \quad P=1$

partie reelle reduite

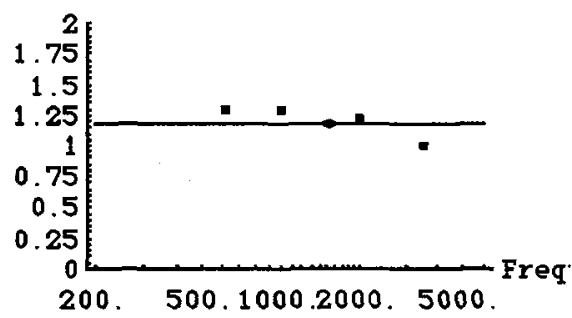

Partie imaginaire reduite

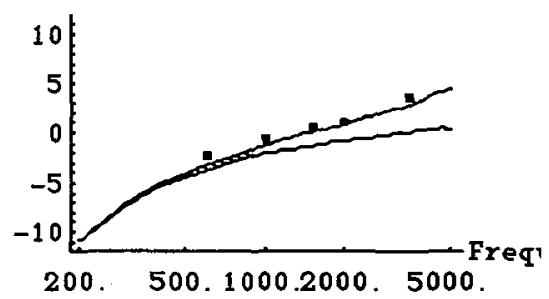

même mousse + tôle perforée $\sigma=4.43 \% \quad h=2 \mathrm{~mm}$

\section{Conclusion}

Le modèle conçu pour les différentes configurations satisfait l'expérience malgré une assez difficile évaluation de la dissipation d'énergie due aux frottements de l'air dans le col que nous retrouvons dans chaque partie réelle. Quant à la partie imaginaire, la méthode de calcul développée rejoint l'expérience. Pour les structures cloisonnées, la longueur corrigée n'est pas rigoureuse car l'impédance de rayonnement d'un piston-plan débouchant dans un cylindre fini n'est pas bien connue. Au terme de cette étude nous sommes parvenus à approcher le modèle concernant les matériaux poreux, avec ou sans tôles perforées. A l'avenir nous étudierons le comportement acoustique et l'efficacité dans le milieu industriel des matériaux stratifiés.

\section{Bibliographie}

[1] UNO Ingard "Perforated facing and sound Absorption" J.A.S.A Mars 1954

[2] Uno Ingard "On the Theory and Design of Acoustic Resonators " J.A.S.A Novembre 1953

[3] P.M.Morse and P.H.Bolt "Acoustic Impedance " Modern Phys 16,92 (1944)

[4] Brillouin "théorie de l'absorption du son sur des panneaux à structures perforés" Cahier du C.T.B $\mathrm{N}^{\circ} 31 \mathrm{pp} 1-15(1951)$ 